\title{
Perturbation Theory for the Decay Rate of Eigenfunctions in the Generalized $N$-body Problem
}

\section{Herbst ${ }^{1}$}

Department of Mathematics, University of Virginia, Charlottesville, VA 22903, USA

Received November 20, 1992

\begin{abstract}
Simple examples are known where eigenfunctions decay faster than the usual upper bounds would lead one to believe. We develop aspects of the perturbation theory of the decay rate of eigenfunctions as measured by radial exponential weights. We show that generically (in a Baire category sense) eigenfunction decay rates are governed by the lowest threshold.
\end{abstract}

\section{Introduction}

There is now an enormous literature on exponential decay of eigenfunctions in the $N$-body problem. The best known upper bonds are due to Agmon [A1]. If $H$ is the generalized $N$-body Schrödinger operator (see Sect. II for details), and $E<\Sigma_{0}(H)=\inf \sigma_{\text {ess }}(H)$, these bounds state that any $L^{2}$ solution of $H \psi=E \psi$ satisfies

$$
|\psi(x)| \leqq c_{\gamma} e^{-\gamma \rho_{A}(x)}, \quad \text { all } \gamma<1,
$$

where $\rho_{A}(x)$ is the distance to the origin in the "Agmon metric" [A1]. If $\psi(x)$ is the unique ground state with eigenvalue $E<\Sigma_{0}(H)$, it is known [CS] that

$$
|\psi(x)| \geqq \tilde{c}_{\gamma} e^{-\gamma \rho_{A}(x)}, \quad \text { all } \gamma>1,
$$

with $\tilde{c}_{\gamma}>0$, but for other eigenfunctions lower bounds are harder to come by. We mention here some results of $[\mathrm{FH} 1,2]$ in this direction: Define

$$
\alpha_{\psi} \equiv \sup \left\{\alpha \geqq 0: \exp (\alpha|x|) \psi \in L^{2}\right\} \text {. }
$$

Then $\alpha_{\psi}^{2}+E$ is a threshold or $+\infty$. (The possibility $+\infty$ can be eliminated with certain assumptions about the potential which we will not make here.) The set $\mathscr{T}(H)$ of thresholds is a closed countable set to be defined later. Suffice it to say for

${ }^{1}$ Supported in part by NSF grant DMS-8807816 
the present that $\min \mathscr{T}(H)=\Sigma_{0}(H)$. We also have the statement (for $E<\Sigma_{0}(H)$ ),

$$
\lim _{r \rightarrow \infty} r^{-1} \ln \left(\int_{S^{n-1}}|\psi(r \omega)|^{2} d \sigma(\omega)\right)^{\frac{1}{2}}=-\alpha_{\psi},
$$

which combines upper and lower bounds. Here $\sigma$ is Lebesgue measure on $S^{n-1}$.

The relationship of the Agmon distance to $\alpha_{\psi}$ is as follows: if we look for the direction of minimal decay given by the Agmon distance, we find

$$
\min _{\omega \in S^{n-1}} \rho_{A}(r \omega)=\left(\sqrt{\Sigma_{0}(H)-E}\right) r .
$$

Thus, if the particular threshold singled out by $\psi$ is $\Sigma_{0}(H)$ in the sense that $\alpha_{\psi}^{2}+E=\Sigma_{0}(H)$, we have $\min _{\omega \in S^{n-1}} \rho_{A}(r \omega)=\alpha_{\psi} r$. The Agmon distance thus gives more information than the upper bound inherent in (1.3) about the behavior of the eigenfunction if the eigenfunction picks out the "right" threshold (namely $\Sigma_{0}(H)$ ). However, this need not be the case (see $\left[\mathrm{FH}_{3} \mathrm{O}_{2}\right]$ for examples), and when the "wrong" threshold is chosen, the Agmon distance may have no relevance to the decay of the eigenfunction. (Of course, the upper bound is always correct but may be very far off from the true behavior.)

Since abnormally rapid decay does indeed occur, two natural problems suggest themselves:

(1) Understand the nature of this decay when it does happen. In particular, find a relation between the decay rate in various directions and spectral data. Aside from (1.3) which only gives information about the rate of decay in the direction of weakest decay, not much is known. (However, see [FH1] for a conjecture and [D1, D2] for some attempts to understand this problem.)

(2) Show that generically (in some sense), the Agmon distance gives the correct decay rate.

In this paper we solve a simplified version of problem (2). We show that generically all eigenfunctions with eigenvalues below $\Sigma_{0}(H)$ pick the "right" threshold, i.e., $\alpha_{\psi}^{2}+E=\Sigma_{0}(H)$. (We will be precise about the word generic in Sect. 3.)

There are two basically different results which need to be proved in our approach. The first, which we accomplish in Sect. 2, is a stability result which in its simplest form says that if $E_{0}<\Sigma_{0}(H)$ is an eigenvalue with corresponding eigenfunctions $\psi_{j}$, each of which has "normal" decay (so that $\alpha_{\psi_{j}}^{2}+E_{0}=\Sigma_{0}(H)$ ), then for small $W \in C_{0}^{\infty}\left(\mathbf{R}^{n}\right)$ the eigenfunctions of $H+W$ with eigenvalues near $E_{0}$ will all have normal decay. (Actually a more general stability result is proved where $W$ is allowed to be a (generalized) $N$-body potential.)

The second result is more perturbation-theoretic and says that if the eigenfunction $\psi$ decays abnormally fast, then we can find an arbitrarily small $W \in C_{0}^{\infty}\left(\mathbf{R}^{n}\right)$ which turns $\psi$ into a normally decaying eigenfunction. Thus even though $\psi$ may be analytic in $W$ (as a vector in $L^{2}$ ), its decay rate can change discontinuously. Our approach to this problem necessitates looking at all orders of perturbation theory. We do this in Sect. 3.

The main results of this paper are summarized in Theorems 2.6, 3.6, and 3.7. There is also a new approach to lower bounds for positive solutions presented in Theorem 3.8. While our method does not reproduce the lower bounds of [CS], the new technique may be of interest. In addition, a new proof of the main result of $[\mathrm{FH} 1]$ is given in an appendix. 
The present paper is similar in structure to [AHS] which develops perturbation theory for embedded eigenvalues. As the reader will see, an eigenvalue with abnormally decaying eigenfunctions is actually an embedded eigenvalue of a certain non-self-adjoint operator and thus the similarity is natural. Indeed, at least in this author's mind, [AHS] was originally conceived as a necessary prerequisite for the present work. In addition to the similarity in structure of the papers, the present paper relies heavily on estimates proved in [AHS]. These estimates originate in [FH1].

The author thanks S. Agmon for useful conversations.

\section{Stability of Normal Exponential Decay}

Throughout this paper,

$$
H=-\Delta+V, \quad V(x)=\sum_{i=1}^{M} v_{i}\left(\pi_{i} x\right),
$$

where the $v_{i}$ are real and $\pi_{i}$ is an orthogonal projection with range $X_{i} \subset \mathbf{R}^{n}$. This type of operator was first introduced by Agmon in [A]. Let $\Delta_{i}=\Delta_{X_{i}}$, the Laplace operator associated to $X_{i}$. We assume

(i) $v_{i}\left(-\Delta_{i}+1\right)^{-1}$ is compact on $L^{2}\left(X_{i}\right)$;

(ii) $\left(-\Delta_{i}+1\right)^{-1} x_{i} \cdot \nabla v_{i}\left(x_{i}\right)\left(-\Delta_{i}+1\right)^{-1}$ is compact on $L^{2}\left(X_{i}\right)$.

Let $\mathscr{L}$ be the family of subspaces $X$ of the form

$$
X=X_{i_{1}}+X_{i_{2}}+\cdots+X_{i_{1}} .
$$

We also include $\{0\}$ and $\mathbf{R}^{n}$ in $\mathscr{L}$. For $X \in \mathscr{L}$ we define $H_{X}=-\Delta_{X}+V_{X}$, where $V_{X}(x)=\sum_{X_{i} \subset X} v_{i}\left(\pi_{i} x\right)$. By convention $H_{\{0\}}=0$ on $\mathscr{C}$. We define $\mathscr{T}(H)=\{\lambda: \lambda$ is an eigenvalue of $H_{X}$ for some $\left.X \neq \mathbf{R}^{n}\right\}$. Thus by definition, $0 \in \mathscr{T}(H)$.

If $\psi$ is an eigenfunction of $H$ with eigenvalue $\lambda_{0}<\Sigma_{0}(H)=\inf \mathscr{T}(H)$, then we say that $\psi$ has normal decay if $\alpha_{\psi}^{2}+\lambda_{0}=\Sigma_{0}(H)$ and abnormal decay otherwise.

We will perturb $H$ by adding a real-valued function $W(x)=\sum_{i=1}^{M} w_{i}\left(\pi_{i} x\right)$, where the $w_{i}$ 's satisfy the same assumptions (2.1) as the $v_{i}$ 's.

We first state a result which essentially says that the number of linearly independent eigenfunctions of $H$ with eigenvalue near $\lambda_{0} \in \mathbf{R}$ which decay at least as fast as $e^{-\alpha r}\left(\alpha^{2}+\lambda_{0} \notin \mathscr{T}(H)\right)$ cannot increase under a small perturbation $W$. The size of $W$ is measured by the norm

$$
|W|_{1}=\left\|W(-\Delta+1)^{-1}\right\|+\left\|(-\Delta+1)^{-1} x \cdot \nabla W(-\Delta+1)^{-1}\right\| .
$$

Theorem 2.1. Suppose $\alpha>0$ and $\alpha^{2}+\lambda_{0} \notin \mathscr{T}(H)$. Then there is an open interval $J$ containing $\lambda_{0}$ and $a \delta>0$ so that if $|W|_{1}<\delta$, then

$$
\sum_{\lambda \in J} \operatorname{dim}\left\{\psi \in \operatorname{ker}(H+W-\lambda): \alpha_{\psi} \geqq \alpha\right\} \leqq \operatorname{dim}\left\{\psi \in \operatorname{ker}\left(H-\lambda_{0}\right): \alpha_{\psi} \geqq \alpha\right\} .
$$

It turns out that this theorem can be thought of as a result about the perturbation of the point spectrum of a certain non-self-adjoint operator. With $\rho_{\alpha}(x)=$ $\exp (\alpha\langle x\rangle),\langle x\rangle=\sqrt{|x|^{2}+1}$, define

$$
H_{\alpha}=H+\nabla \ln \rho_{\alpha} \cdot D+D \cdot \nabla \ln \rho_{\alpha}-\left|\nabla \ln \rho_{\alpha}\right|^{2}, \quad \mathscr{D}\left(H_{\alpha}\right)=\mathscr{D}(\Delta),
$$


where $D$ is the gradient operator. Note that $H_{\alpha}$ is the closure of $\rho_{\alpha} H \rho_{\alpha}^{-1} \mid C_{0}^{\infty}\left(\mathbf{R}^{n}\right)$.

Theorem 2.2. Suppose $\alpha>0$ and $\alpha^{2}+\lambda_{0} \notin \mathscr{T}(H)$. Then there is an open interval $J$ containing $\lambda_{0}$ and $a \delta>0$ so that if $|W|_{1}<\delta$, then

$$
\sum_{\lambda \in J} \operatorname{dim} \operatorname{ker}\left(H_{\alpha}+W-\lambda\right) \leqq \operatorname{dim} \operatorname{ker}\left(H_{\alpha}-\lambda_{0}\right)
$$

Deduction of Theorem 2.1 from Theorem 2.2. It clearly suffices to prove (i) and (ii) below:

(i) There is a $\delta>0$ so that if $\lambda_{0}+\alpha^{2} \notin \mathscr{T}(H)$, then $\left|\lambda-\lambda_{0}\right|+|W|_{1}<\delta$ implies $\lambda+\alpha^{2} \notin \mathscr{T}(H+W)$, and

(ii) If $\lambda+\alpha^{2} \notin \mathscr{T}(H)$, then $\operatorname{dim} \operatorname{ker}\left(H_{\alpha}-\lambda\right)=\operatorname{dim}\left\{\psi \in \operatorname{ker}(H-\lambda): \alpha_{\psi} \geqq \alpha\right\}$.

To prove (i) note that if $X \in \mathscr{L}$ and $X \neq \mathbf{R}^{n}$, then $\lambda_{0}+\alpha^{2} \notin \mathscr{T}\left(H_{X}\right) \cup \sigma_{p}\left(H_{X}\right)$. According to Theorem 2.5 in [AHS], if $\left|\lambda-\lambda_{0}\right|+|W|_{1}$ is small enough, $\lambda+\alpha^{2} \notin \sigma_{p}\left((H+W)_{X}\right)$. Hence, if $\left|\lambda-\lambda_{0}\right|+|W|_{1}$ is small enough $\lambda+\alpha^{2} \notin \mathscr{T}(H+W)$. To prove (ii) we show that if $\lambda+\alpha^{2} \notin \mathscr{T}(H)$, then $\operatorname{ker}\left(H_{\alpha}-\lambda\right)=\rho_{\alpha}\left(\operatorname{ker}(H-\lambda) \cap\left\{\psi: \alpha_{\psi} \geqq \alpha\right\}\right)$. Thus, suppose $f \in \operatorname{ker}\left(H_{\alpha}-\lambda\right)$. Then let $\psi=\rho_{\alpha}^{-1} f$. Formally we have $0=\left(\rho_{\alpha} H \rho_{\alpha}^{-1}-\lambda\right) f=\rho_{\alpha}(H-\lambda) \rho_{\alpha}^{-1} f$ so that $\psi \in \operatorname{ker}(H-\lambda)$ and $\rho_{\alpha} \psi \in L^{2}$, and thus $\alpha_{\psi} \geqq \alpha$. This is easily made into a proof. Suppose $\phi \in C_{0}^{\infty}\left(\mathbf{R}^{n}\right)$. Then we easily see that

$$
0=\left(\rho_{\alpha}^{-1}(H-\lambda) \rho_{\alpha} \phi, f\right)=\left((H-\lambda) \rho_{\alpha} \phi, \rho_{\alpha}^{-1} f\right) .
$$

But $\rho_{\alpha}^{-1} \mathscr{D}(\Delta) \subset \mathscr{D}(\Delta)$ so that

$$
\left(\rho_{\alpha} \phi,(H-\lambda) \psi\right)=0
$$

for all $\phi \in C_{0}^{\infty}\left(\mathbf{R}^{n}\right)$. Thus $\psi \in \operatorname{ker}(H-\lambda)$.

If $\psi \in \operatorname{ker}(H-\lambda)$ and $\alpha_{\psi} \geqq \alpha$, then since by [FH1], $\alpha_{\psi}^{2}+\lambda \in \mathscr{T}(H) \cup\{\infty\}$, while $\alpha^{2}+\lambda \notin \mathscr{T}(H)$ we have $\alpha_{\psi}>\alpha$. In particular, $f=\rho_{\alpha} \psi \in L^{2}$. It follows easily that $\rho_{\alpha} \psi \in \mathscr{D}(\Delta)$ and $H_{\alpha} f=\lambda f$. Hence $\psi=\rho_{\alpha}^{-1} f$ with $f \in \operatorname{ker}\left(H_{\alpha}-\lambda\right)$.

We now embark on a proof of Theorem 2.2: From Theorem 3.1 of [AHS] we have for $\left|\lambda-\lambda_{0}\right|+|W|_{1}<\delta_{1}$,

$$
k\left\|\langle x\rangle^{\frac{1}{2}} \rho_{\alpha}(H+W-\lambda) \phi\right\| \geqq\left\|\rho_{\alpha} \phi\right\|-\left\|K \rho_{\alpha} \phi\right\|,
$$

with some compact operator $K$ independent of $W$ and $\lambda$ and for all $\phi \in C_{0}^{\infty}\left(\mathbf{R}^{n}\right)$. Let $\chi_{R}$ be the characteristic function of $\{x:|x|<R\}$. Then for large enough $R$,

$$
\left\|K\left(1-\chi_{R}\right)\right\|<\frac{1}{2}
$$

so that

$$
\left\|K \rho_{\alpha} \phi\right\| \leqq \frac{1}{2}\left(\left\|\rho_{\alpha} \phi\right\|+c\|\phi\|\right),
$$

and thus $(2.2 \mathrm{a})$ implies

$$
k^{\prime}\left\|\langle x\rangle^{\frac{1}{2}} \rho_{\alpha}(H+W-\lambda) \phi\right\| \geqq\left\|\rho_{\alpha} \phi\right\|-c\|\phi\| .
$$

It follows easily from $(2.2 \mathrm{a})$ that

$$
k\left\|\langle x\rangle^{\frac{1}{2}}\left(H_{\alpha}+W-\lambda\right) f\right\| \geqq\|f\|-\|K f\|, \quad f \in \mathscr{D}(\Delta) .
$$


It is important to note that (2.2b) is claimed to hold only for $\phi \in C_{0}^{\infty}\left(\mathbf{R}^{n}\right)$. While (2.2b) has a meaning for $\phi \in \mathscr{D}(H)=\mathscr{D}(\Delta)$, where both right and left sides may be infinite, it is often incorrect even though it makes sense. Indeed, in Proposition 3.5 it is shown that under certain assumptions (2.2b) cannot hold for all $\phi \in \mathscr{D}(H)$.

Let $P_{0}$ be the orthogonal projection onto the subspace of all $\psi \in \operatorname{ker}\left(H-\lambda_{0}\right)$ with $\alpha_{\psi} \geqq \alpha$. As in the proof of Theorem 2.1, $\alpha_{\psi}>\alpha$. It follows from (2.3) that $P_{0}$ has finite rank for otherwise we could argue that for an orthonormal sequence $\left\{f_{m}: m=1,2, \ldots\right\}$ of eigenfunctions of $H_{\alpha}$ we had $\left\|K f_{m}\right\| \geqq\left\|f_{m}\right\|$. (Note that we have not assumed that $\lambda_{0} \notin \mathscr{T}(H)$ so that as far as we know there is nothing to prevent $\operatorname{ker}\left(H-\lambda_{0}\right)$ from being infinite dimensional.) It follows that $P_{\alpha}=\rho_{\alpha} P_{0} \rho_{\alpha}^{-1}$ is a bounded finite rank projection onto $\operatorname{ker}\left(H_{\alpha}-\lambda_{0}\right)$.

We claim that for some $\delta>0$ and $\bar{k}>0$ we have

$$
\bar{k}\left\|\langle x\rangle^{\frac{1}{2}}\left(H_{\alpha}+W-\lambda\right)\left(1-P_{\alpha}\right) f\right\| \geqq\left\|\left(1-P_{\alpha}\right) f\right\|
$$

for all $f \in \mathscr{D}(\Delta)$ and $\left|\lambda-\lambda_{0}\right|+|W|_{1}<\delta$. Suppose not. Then there is a sequence $f_{n} \in \mathscr{D}(\Delta)$ with $P_{\alpha} f_{n}=0$ and $\lambda_{n} \rightarrow \lambda_{0},\left|W_{n}\right|_{1} \rightarrow 0$ so that $\left\|f_{n}\right\|=1$ while $\left\|\langle x\rangle^{\frac{1}{2}}\left(H_{\alpha}+W_{n}-\lambda_{n}\right) f_{n}\right\| \rightarrow 0$. We can assume that $f_{n} \stackrel{w}{\rightarrow} f$. It follows that for $\phi \in C_{0}^{\infty}\left(\mathbf{R}^{n}\right)$

$$
0=\lim \left(\phi,\left(H_{\alpha}+W_{n}-\lambda_{n}\right) f_{n}\right)=\left(\left(H_{\alpha}^{*}-\lambda_{0}\right) \phi, f\right),
$$

which implies $\left(H_{\alpha}-\lambda_{0}\right) f=0$. We also have $0=P_{\alpha} f_{n} \rightarrow P_{\alpha} f$ so that $\rho_{\alpha}^{-1} f$ is orthogonal to Range $P_{0}$ but also in Range $P_{0}$. Hence $f=0$. From (2.3) it now follows that $0 \geqq 1$, and thus for some $\delta>0(2.4)$ holds for some $\bar{k}>0$ if $\left|\lambda-\lambda_{0}\right|+|W|_{1}<\delta$. Now suppose

$$
\sum_{\lambda \in J} \operatorname{dim} \operatorname{ker}\left(H_{\alpha}+W-\lambda\right)>\operatorname{dim} \operatorname{ker}\left(H_{\alpha}-\lambda_{0}\right) \equiv L .
$$

Let $f_{j} \in \operatorname{ker}\left(H_{\alpha}+W-\lambda_{j}\right), \lambda_{j} \in J, j=1, \ldots, L+1$, and $f=\sum_{j=1}^{L+1} \beta_{j} f_{j}$ with $P_{\alpha} f=0$. We an assume that the $\rho_{\alpha}^{-1} f_{j}=\psi_{j}, j=1, \ldots, L+1$ are orthonormal, and that $\psi=\rho_{\alpha}^{-1} f$ has norm 1 . Then

$$
\bar{k}\left\|\langle x\rangle^{\frac{1}{2}}\left(H_{\alpha}+W-\lambda_{0}\right) f\right\| \geqq\|f\|,
$$

and

$$
\left\|\langle x\rangle^{\frac{1}{2}}\left(H_{\alpha}+W-\lambda_{0}\right) f\right\| \leqq \sum_{j}\left|\beta_{j}\right|\left|\lambda_{0}-\lambda_{j}\right|\left\|\langle x\rangle^{\frac{1}{2}} f_{j}\right\|
$$

and thus

$$
\bar{k}(L+1)|J| \sup _{j}\left\|\langle x\rangle^{\frac{1}{2}} f_{j}\right\| \geqq 1,
$$

where $|J|=$ length of $J$. We can use (2.2b) with $\phi=\psi_{j}, \lambda=\lambda_{j}$, and $\alpha$ replaced by a slightly larger number $\beta$ to conclude that $\left\|\rho_{\beta} \psi_{j}\right\| \leqq c\left\|\psi_{j}\right\|=c$ so that

$$
\sup _{j}\left\|\langle x\rangle^{\frac{1}{2}} f_{j}\right\|=\sup _{j}\left\|\langle x\rangle^{\frac{1}{2}} \rho_{\alpha} \rho_{\beta}^{-1} \rho_{\beta} \psi_{j}\right\| \leqq c^{\prime}
$$

Thus

$$
\bar{k}(L+1)|J| c^{\prime} \geqq 1,
$$


which is a contradiction if $|J|$ is small enough.

We now discuss the meaning of Theorem 2.1 further. It is easy to see that $\Sigma_{0}(H+W)$ is continuous in the variable $W$. In addition, $\Sigma_{0}(H)$ is always an isolated point of $\mathscr{T}(H)$. This follows from a theorem of Perry [P] which says that under our assumptions eigenvalues can only accumulate at thresholds from below. Thus if we let

$$
\Sigma_{1}(H)=\inf \left[\mathscr{T}(H) \cap\left(\Sigma_{0}(H), \infty\right) \cup\{\infty\}\right]
$$

we have

$$
\Sigma_{1}(H)>\Sigma_{0}(H) .
$$

We have allowed for the possibility that $\mathscr{T}(H) \cap\left(\Sigma_{0}(H), \infty\right)$ is empty in which case $\Sigma_{1}(H)=+\infty$.

Suppose now that $\lambda_{0}$ is an eigenvalue of $H$ with $\lambda_{0}<\Sigma_{0}(H)$. The question which we want to address is how shall we choose $\alpha^{2}+\lambda_{0}$ in order to get information from Theorem 2.1? If $\alpha^{2}+\lambda_{0}<\Sigma_{0}(H)$ no information is provided. This is because for $\left|\lambda-\lambda_{0}\right|+|W|_{1}$ small we will have $\alpha^{2}+\lambda<\Sigma_{0}(H+W)$. But $\alpha_{\psi}^{2}+\lambda \geqq \Sigma_{0}(H+W)$ for all $\psi \in \operatorname{ker}(H+W-\lambda)$. The most obvious choice to take is

$$
\Sigma_{0}(H)<\alpha^{2}+\lambda<\Sigma_{1}(H) .
$$

We shall do this but we must point out that even though we always have $\Sigma_{1}(H+W)-\Sigma_{0}(H+W)>0$, there are potentials $V$ where this difference may be arbitrarily small for arbitrarily small $W$.

In such a situation it is impossible to have $\alpha^{2}+\lambda$ between $\Sigma_{0}(H+W)$ and $\Sigma_{1}(H+W)$ for all small $W$.

There are two special conditions which produce a situation where it is possible to have $\left|W_{n}\right|_{1} \rightarrow 0$ while $\Sigma_{1}\left(H+W_{n}\right)-\Sigma_{0}\left(H+W_{n}\right) \rightarrow 0$. In order to discuss them we make two definitions. In the following $X<Y$ means $X \subset Y$ and $X \neq Y$, and $\sigma_{p}(A)$ is the set of eigenvalues of $A$.

Definition 2.3. We say that $v$ is critical for subsystems if there is an $X \in \mathscr{L}$ with $0<X<\mathbf{R}^{n}$ such that $\Sigma_{0}(H)=\Sigma_{0}\left(H_{X}\right) \notin \sigma_{p}\left(H_{X}\right)$ and given $\varepsilon>0$ there is $a$ and an eigenvalue $\lambda(W)$ of $(H+W)_{X}$ such that $|W|_{1}<\varepsilon$ and $\left|\lambda(W)-\Sigma_{0}\left((H+W)_{X}\right)\right|<\varepsilon$.

Clearly, if $V$ is critical for subsystems an eigenvalue is just about to emerge from the bottom of the essential spectrum of one of the $H_{X}$ with $0<X<\mathbf{R}^{n}$ and $\sigma\left(H_{X}\right)=\sigma_{\text {ess }}\left(H_{X}\right)$. This situation has been studied for certain kinds of potentials [KS1, 2; Ka; S2].

Another condition which may cause $\Sigma_{1}(H+W)-\Sigma_{0}(H+W)$ to become arbitrarily small for small $W$ is degeneracy.

Definition 2.4. We say that $\Sigma_{0}(H)$ is degenerate $($ in $\mathscr{T}(H))$ if there are two subspaces $X_{1}, X_{2} \in \mathscr{L}$ with $X_{1} \neq X_{2}$ and both $<\mathbf{R}^{n}$ such that $\Sigma_{0}(H)$ is an eigenvalue of both $H_{X_{1}}$ and $H_{X_{2}}$.

In this case clearly a small perturbation $W$ can split $\Sigma_{0}$ into $\Sigma_{0}$ and $\Sigma_{1}$ which will be arbitrarily close.

Proposition 2.5. Suppose $\Sigma_{0}(H)$ is not degenerate in $\mathscr{T}(H)$ and $V$ is not critical for subsystems. Then there are positive numbers $\delta_{1}$ and $\delta_{2}$ so that if $|W|_{1}<\delta_{1}$, then

$$
\Sigma_{1}(H+W)-\Sigma_{0}(H+W)>\delta_{2} .
$$


Proof. Suppose $\Sigma_{0}(H)<0$. Then $\Sigma_{0}(H) \in \sigma_{p}\left(H_{X_{0}}\right)$ for exactly one $X_{0}<\mathbf{R}^{n}$. Consider the set

$$
\mathscr{L}_{0}=\left\{X \in \mathscr{L}: 0<X<\mathbf{R}^{n}, \Sigma_{0}(H)=\Sigma_{0}\left(H_{X}\right)\right\} .
$$

If $X \in \mathscr{L}_{0}$, then since $\Sigma_{0}(H) \in \mathscr{T}\left(H_{X}\right)$ there must be a $Y \in \mathscr{L}$ with $Y<X$ such that $\Sigma_{0}(H) \in \sigma_{p}\left(H_{Y}\right)$. It follows from the nondegeneracy condition that $X_{0} \notin \mathscr{L}_{0}$ and that $\Sigma_{0}(H) \in \sigma_{\text {disc }}\left(H_{X_{0}}\right)$. Suppose $X \in \mathscr{L}_{0}$. Then again, by the nondegeneracy condition we cannot have $\Sigma_{0}(H) \in \sigma_{p}\left(H_{X}\right)$. Since $V$ is not critical for subsystems there is a neighborhood $N_{X}$ of $\Sigma_{0}(H)$ and a positive number $\delta_{X}$ so that $N_{X} \cap \sigma_{p}\left((H+W)_{X}\right)=\emptyset$ if $|W|_{1}<\delta_{X}$. If $X$ is in

$$
\left\{X \in \mathscr{L} \backslash\left\{X_{0}\right\}: 0<X<\mathbf{R}^{n}, \Sigma_{0}(H)<\Sigma_{0}\left(H_{X}\right)\right\},
$$

clearly the same is true. Similarly, if $X=\{0\}$. Finally, for small $W$ there is a neighborhood $N_{X_{0}}$ of $\Sigma_{0}(H)$ so that $N_{X_{0}} \cap \sigma_{p}\left((H+W)_{X_{0}}\right)=\left\{\inf \sigma\left((H+W)_{X_{0}}\right)\right\}$ and by continuity, for small $W, \Sigma_{0}(H+W)=\inf \sigma\left((H+W)_{X_{0}}\right)$. Hence if $\Sigma_{0}(H)<0$ we have shown that for small $W, \Sigma_{1}(H+W)-\Sigma_{0}(H+W)$ is bounded away from zero.

Suppose $\Sigma_{0}(H)=0$. This situation is easier to deal with. By the nondegeneracy condition (note that 0 is an eigenvalue of $H_{\{0\}}$ ), if $0<X<\mathbf{R}^{n}$ and $X \in \mathscr{L}$, Then $\Sigma_{0}(H)=0 \notin \sigma_{p}\left(H_{X}\right)$. Because $V$ is not critical for subsystems, there is a neighborhood $N_{X}$ of 0 and a positive number $\delta_{X}$ so that $N_{X} \cap \sigma_{p}\left((H+W)_{X}\right)=\emptyset$ if $|W|_{1}<\delta_{X}$. Thus for small $|W|_{1}, \Sigma_{0}(H+W)=0$ and $\Sigma_{1}(H+W)$ is bounded away from zero.

Returning to our main line of development, we note that if $\Sigma_{0}(H)$ is nondegenerate in $\mathscr{T}(H)$ and $V$ is not critical for subsystems we can choose $\alpha>0$ so that $\Sigma_{0}(H+W)<\alpha^{2}+\lambda<\Sigma_{1}(H+W)$ for $|W|_{1}+\left|\lambda-\lambda_{0}\right|$ small. We then learn from Theorem 2.1 that the number of abnormally decaying eigenfunctions with eigenvalue near $\lambda_{0}$ cannot increase. In particular, if all eigenfunctions of $H$ at $\lambda_{0}$ have normal decay, the same is true for the eigenfunctions of $H+W$ at $\lambda$ if $|W|_{1}+\left|\lambda-\lambda_{0}\right|$ is sufficiently small. This is summarized in Theorem 2.6 below.

If we have degeneracy or criticality there can be at least two thresholds $\Sigma_{0}$ and $\Sigma_{1}$ emerging from a point as $W$ is turned on, and an eigenfunction with eigenvalue initially at $\lambda_{0}$, and with $\alpha_{\psi}^{2}+\lambda_{0}=\Sigma_{0}(H)$ has a choice of which threshold to pick to control its decay rate, a choice from among at least two thresholds emerging from the same point. We cannot prove that the eigenfunction always picks $\Sigma_{0}$, and in fact we believe that it does not. What we can prove is stated in the next theorem.

Theorem 2.6. Suppose $V$ is not critical for subsystems, that $\Sigma_{0}(H)$ is nondegenerate in $\mathscr{T}(H)$, and that $\lambda_{0}<\Sigma_{0}(H)$. Then there is $a \delta>0$ and an open interval $J$ containing $\lambda_{0}$ so that if $|W|_{1}<\delta$, then

$$
\begin{gathered}
\sum_{\lambda \in J} \operatorname{dim}\left\{\psi \in \operatorname{ker}(H+W-\lambda): \alpha_{\psi}^{2}+\lambda>\Sigma_{0}(H+W)\right\} \\
\leqq \operatorname{dim}\left\{\psi \in \operatorname{ker}\left(H-\lambda_{0}\right): \alpha_{\psi}^{2}+\lambda_{0}>\Sigma_{0}(H)\right\} .
\end{gathered}
$$

Proof. We choose $\alpha>0$ so that $\Sigma_{0}(H)<\alpha^{2}+\lambda_{0}<\Sigma_{1}(H)$. We can choose $\delta$ and $|J|$ small enough so that $\Sigma_{0}(H+W)<\alpha^{2}+\lambda<\Sigma_{1}(H+W)$ if $\lambda \in J$ and $|W|_{1}<\delta$. Then $\operatorname{dim}\left\{\psi \in \operatorname{ker}(H+W-\lambda): \alpha_{\psi} \geqq \alpha\right\}=\operatorname{dim}\{\psi \in \operatorname{ker}(H+W-\lambda):$ $\left.\alpha_{\psi}^{2}+\lambda>\Sigma_{0}(H+W)\right\}$, and the result follows from Theorem 2.1. 
Remark. If we restrict $W$ to a subclass so that $\Sigma_{1}(H+W)-\Sigma_{0}(H+W) \geqq c>0$ for $W$ in this subclass and $|W|_{1}$ small, clearly the conclusion of the theorem holds even if $V$ does not satisfy the assumptions of Proposition 2.5. This happens, for example, if $W(-\Delta+1)^{-1}$ is compact for then $\mathscr{T}(H+W)=\mathscr{T}(H)$.

\section{Perturbation Theory}

In this section we will consider an eigenfunction $\phi_{0}$ of $H$ with eigenvalue $\lambda_{0}<\Sigma_{0}(H)$. Under the assumption that $\phi_{0}$ has abnormal decay, we will show that an arbitrarily small perturbation $W \in C_{0}^{\infty}\left(\mathbf{R}^{n}\right)$ can be added to $H$ to turn $\phi_{0}$ into a normally decaying eigenfunction. This result, along with the stability result of Sect. 2, will be enough to show that generically all eigenfunctions with eigenvalue below $\Sigma_{0}(H)$ have normal decay.

A byproduct of our methods is a new approach to lower bounds for positive eigenfunctions. At present, due to the use of radial weights, this approach cannot reproduce the results of Carmona and Simon [CS], but it may be of interest nevertheless.

In this section we will always assume (2.1), but we will sometimes also need the following:

For each $i,\langle y\rangle^{\varepsilon} v_{i}(y)\left(-\Delta_{i}+1\right)^{-1}$ is bounded for some $\varepsilon>0$.

Consider the situation where a non-degenerate isolated eigenvalue of $H$ has a corresponding eigenfunction with abnormal decay. We then add a small perturbation to $H$ to obtain $H+t W$ with corresponding eigenfunction $\phi(t)$. Our objective is to choose $W$ so that $\phi(t)$ has normal decay for all small $|t|>0$. The next result shows we need only make sure that some derivative $\phi^{(k)}(0)=d^{k} \phi(t) /\left.d t^{k}\right|_{t=0}$ has normal decay.

Proposition 3.1. Suppose $W(x)=\sum_{i=1}^{M} w_{i}\left(\pi_{i} x\right)$ satisfies (2.1), and that for $t$ in an open interval I containing 0 ,

$$
(H+t W-\lambda(t)) \phi(t)=0,
$$

where we assume $\phi(\cdot)$ and $\lambda(\cdot)$ are $C^{\infty}$ functions on I. Suppose $\beta_{0}>0$ is chosen so that $\beta_{0}^{2}+\lambda(0) \notin \mathscr{T}(H)$ and $\alpha_{\phi(0)}>\beta_{0}$ but for some $k \geqq 1, \alpha_{\phi^{(k)}(0)}<\beta_{0}$. Then there is an open interval $J$ containing 0 with $J \subset I$ such that for all $t \in J \backslash\{0\}$,

$$
\alpha_{\phi(t)}<\beta_{0} .
$$

Proof. Differentiating (3.2) $m+1$ times gives

$$
\begin{aligned}
\left(H-\lambda_{0}\right) \phi^{(m+1)}(0)= & (m+1)\left(\lambda^{(1)}(0)-W\right) \phi^{(m)}(0) \\
& +\sum_{k=2}^{m+1}\left(\begin{array}{c}
m+1 \\
k
\end{array}\right) \lambda^{(k)}(0) \phi^{(m+1-k)}(0),
\end{aligned}
$$

where $\lambda_{0}=\lambda(0)$. Let $l=\min \left\{k \geqq 0: \alpha_{\phi^{(k)}(0)}<\beta_{0}\right\}$. Using (3.3), it follows by induction and the methods of $[\mathrm{FH} 1]$ or the Appendix, that for $0 \leqq k \leqq l$, we have $\alpha_{\phi^{(k)}(0)}>\beta_{0}$.

Suppose the proposition is false. Then there is a sequence $t_{j} \rightarrow 0\left(t_{j} \neq 0\right)$ with $\alpha_{\phi\left(t_{j}\right)}>\beta_{0}$. This follows from the proof of Theorem 2.1 which shows that for small $|t|, \alpha_{\phi(t)} \neq \beta_{0}$. 
Given any $f \in C^{\infty}(I)$, let

$$
f_{[j]}(t) \equiv f(t)-\sum_{k=0}^{j} \frac{f^{(k)}(0)}{k !} t^{k} .
$$

For $f$ and $g \in C^{\infty}(I)$ we will make use of the easy proved formula

$$
(f g)_{[j]}(t)=f(t) g_{[j]}(t)+\sum_{k=0}^{j} f_{[k]}(t) g^{(j-k)}(0) \frac{t^{j-k}}{(j-k) !} .
$$

We take $f(t)=H+t W-\lambda(t)$, and $g(t)=\phi(t)$, and find (using $\left.(f g)_{[j]}(t) \equiv 0\right)$,

$$
\begin{aligned}
(H+t W-\lambda(t)) \phi_{[l-1]}(t)= & (\lambda(t)-\lambda(0)-t W) \phi^{(l-1)}(0) t^{l-1} /(l-1) ! \\
& +\sum_{j=1}^{l-1} \lambda_{[j]}(t) \phi^{(l-1-j)}(0) \frac{t^{l-1-j}}{(l-1-j) !} .
\end{aligned}
$$

We denote the right side of (3.5) divided by $t^{l}$ by $f_{l}(t)$ and write $t^{-l} \phi_{[l-1]}(t)=\psi_{l}(t)$. We thus have

$$
(H+t W-\lambda(t)) \psi_{l}(t)=f_{l}(t) .
$$

We know that $\psi_{l}\left(t_{j}\right)=\psi_{j}$ satisfies $\alpha_{\psi_{j}}>\beta_{0}$ and thus use of $\psi_{j}$ in (2.2b) is easily justified. We find

$$
k^{\prime}\left\|\langle x\rangle^{\frac{1}{2}} \rho_{\beta_{0}} f_{l}\left(t_{j}\right)\right\| \geqq\left\|\rho_{\beta_{0}} \psi_{l}\left(t_{j}\right)\right\|-c\left\|\psi_{l}\left(t_{j}\right)\right\| .
$$

But $\lim _{t \rightarrow 0} \psi_{l}(t)=\phi^{(l)}(0) / l$ ! and $\lambda_{[j]}(t) / t^{j+1} \rightarrow \lambda^{(j+1)}(0) /(j+1)$ ! This shows that $\left\|\psi_{l}\left(t_{j}\right)\right\|$ and the left side of (3.6) are uniformly bounded so that

$$
\lim _{j \rightarrow \infty} \sup \left\|\rho_{\beta_{0}} \psi_{l}\left(t_{j}\right)\right\|<\infty .
$$

As a consequence, $\left\|\rho_{\beta_{0}} \phi^{(l)}(0)\right\|<\infty$, and thus $\alpha_{\phi^{(l)}(0)} \geqq \beta_{0}$, a contradiction.

Corollary 3.2. Suppose $\lambda_{0}<\Sigma_{0}(H)$ is a non-degenerate eigenvalue of $H=-\Delta+V$, where $V$ satisfies (2.1). Suppose $W$ is a real function in $C_{0}^{\infty}\left(\mathbf{R}^{n}\right)$, and that $\phi(t)$ is a smooth normalized eigenfunction of $H+t W$ for small $|t|$ with $\left(H-\lambda_{0}\right) \phi(0)=0$, whose existence is guaranteed by standard perturbation theory. Suppose $\alpha_{\phi(0)}>\sqrt{\Sigma_{0}(H)-\lambda_{0}}$ but for some $k>0, \alpha_{\phi^{(k)}(0)}=\sqrt{\Sigma_{0}(H)-\lambda_{0}}$. Then for all small $|t|>0$,

$$
\alpha_{\phi(t)}=\sqrt{\Sigma_{0}(H)-\lambda(t)},
$$

where $(H+t W-\lambda(t)) \phi(t)=0$.

Proof. Just choose $\beta_{0}>0$ with $\Sigma_{0}(H)<\beta_{0}^{2}+\lambda_{0}<\Sigma_{1}(H)$ in Proposition 3.1.

Note that it follows from (3.3) and the Appendix that $\alpha_{\phi^{(k)}(0)} \geqq \sqrt{\Sigma_{0}(H)-\lambda_{0}}$ for all $k$. We now allow ourselves to contemplate the possibility that no matter what real $W \in C_{0}^{\infty}\left(\mathbf{R}^{n}\right)$ we choose, we obtain $\alpha_{\phi^{(k)}(0)}>\sqrt{\Sigma_{0}(H)-\lambda_{0}}$. The proof of the following proposition is somewhat involved.

Proposition 3.3. Suppose $H=-\Delta+V$, where $V$ satisfies (2.1) and that $\lambda_{0}<\Sigma_{0}(H)$ is a non-degenerate eigenvalue of $H$ with eigenfunction $\phi_{0}$ satisfying $\alpha_{\phi_{0}}>\sqrt{\Sigma_{0}(H)-\lambda_{0}}$. Suppose that for each real $W \in C_{0}^{\infty}\left(\mathbf{R}^{n}\right)$ we have

$$
\alpha_{\phi^{(k)}(0)}>\sqrt{\Sigma_{0}(H)-\lambda_{0}}, \quad \text { all } k \geqq 1 \text {. }
$$


Let $Q$ be a real function in $C_{0}^{\infty}\left(\mathbf{R}^{n}\right)$ so that $\lambda_{0} \notin \sigma(H+Q)$. Then for every $\beta>0$ satisfying

$$
\Sigma_{0}(H)<\beta^{2}+\lambda_{0}<\Sigma_{1}(H),
$$

there is a constant $k(\beta)>0$ so that for all $\psi \in \mathscr{D}(\mathscr{H})$,

$$
k(\beta)\left\|\langle x\rangle^{\frac{1}{2}} \rho_{\beta}\left(H+Q-\lambda_{0}\right) \psi\right\| \geqq\left\|\rho_{\beta} \psi\right\| .
$$

We remark that the significance of this result is that (3.7) holds for all $\psi \in \mathscr{D}(H)$. We will see that under a mild additional assumption on the potentials, namely (3.1), this cannot be.

Proof. We assume that $\left\|\phi_{0}\right\|=1$ and define $P_{0}$ as the projection $P_{0} f=\left(\phi_{0}, f\right) \phi_{0}$. Since many $W$ 's in $C_{0}^{\infty}\left(\mathbf{R}^{n}\right)$ will appear in the proof we put a subscript on $\phi(t)$ and $\lambda(t)$ so that

$$
\left(H+t W-\lambda_{W}(t)\right) \phi_{W}(t)=0, \quad \phi_{W}(0)=\phi_{0}, \quad \lambda_{W}(0)=\lambda_{0},
$$

where $W$ is a real function in $C_{0}^{\infty}\left(\mathbf{R}^{n}\right)$, and $t$ is in some small $W$-dependent interval containing zero. We remind the reader that $\lambda_{W}^{(1)}(0)=\left(\phi_{0}, W \phi_{0}\right)$.

The main part of the proof consists of accumulating large numbers of functions $f$ in the range of $H+P_{0}-\lambda_{0}$ so that inequalities similar to (3.7) hold for $\psi=G f$, $G=\left(H+P_{0}-\lambda_{0}\right)^{-1}$. Namely we would like to prove

$$
k(\beta)\left\|\langle x\rangle^{\frac{1}{2}} \rho_{\beta}\left(H+P_{0}-\lambda_{0}\right) \psi\right\| \geqq\left\|\rho_{\beta} \psi\right\|
$$

for such $\psi$. For this purpose, let

$$
\operatorname{Ran}_{m}=\operatorname{span}\left\{\left(H+P_{0}-\lambda_{0}\right) \phi_{W}^{(m)}(0): W \text { is real and in } C_{0}^{\infty}\left(\mathbf{R}^{n}\right)\right\} \text {. }
$$

For any set $S \subset L^{2}\left(\mathbf{R}^{n}\right)$, let

$$
\begin{aligned}
\tilde{S}= & S \cup\left\{f \in L^{2}\left(\mathbf{R}^{n}\right) \text { : for any ball } B \text { contered at } 0\right. \\
& \text { there is a sequence } f_{j} \in S \text { with }\left\|\rho_{\beta}\left(f_{j}-\chi_{B} f\right)\right\| \rightarrow 0 \\
& \text { for all } \left.\beta \text { with } \beta^{2}+\lambda_{0}<\Sigma_{1}(H)\right\},
\end{aligned}
$$

where $\chi_{B}$ is the indicator function of $B$.

Starting with $\mathscr{D}_{0}=\left\{\mu \phi_{0}: \mu \in \mathbf{C}\right\}$, let

$$
\mathscr{D}_{m+1}=\tilde{\mathscr{D}}_{m}+\operatorname{Ran}_{m+1} .
$$

We claim there is an increasing sequence of Borel sets $\left\{A_{j}\right\}$ such that for $m \geqq 1$, $\tilde{\mathscr{D}}_{m}=L^{2}\left(A_{m}\right)$. (By defintion, $L^{2}(A)=\left\{f \in L^{2}\left(\mathbf{R}^{n}\right): f=0\right.$ a.e. on $\left.A^{c}\right\}$.) In fact, we will show that for $m \geqq 1$,

$$
\tilde{\mathscr{D}}_{m+1}=\text { closure } \operatorname{span}\left\{f G h_{1}+h_{2}: f \in L^{\infty}\left(\mathbf{R}^{n}\right), h_{1} \text { and } h_{2} \in L^{2}\left(A_{m}\right)\right\} .
$$

Let us compute $\tilde{\mathscr{D}}_{1}$. Specializing (3.3) to $m=0$ we see that

$$
\mathscr{D}_{1}=\left\{(W+\mu) \phi_{0}: W \in C_{0}^{\infty}\left(\mathbf{R}^{n}\right), \mu \in \mathbf{C}\right\} .
$$

Setting $N=\left\{x: \phi_{0}(x)=0\right\}$, we easily find $\tilde{\mathscr{D}}_{1}=L^{2}\left(N^{c}\right)$, and thus we can take $A_{1}=N^{c}$. If we knew that meas $(N)=0$, It would immediately follow that $\mathscr{\mathscr { D }}_{m}=L^{2}\left(\mathbf{R}^{n}\right)$ for all $m \geqq 1$, but meas $(N)=0$ is not known at present unless strong assumptions are made about the interactions $v_{i}$.

Given that (3.8) holds for some $m$, it easily follows that $\tilde{\mathscr{D}}_{m+1}=L^{2}\left(A_{m+1}\right)$ for some $A_{m+1} \supset A_{m}$. We give the proof. Suppose $h$ is orthogonal to $\mathscr{D}_{m+1}$. Then 
clearly $h \in L^{2}\left(A_{m}^{c}\right)$ and $\left(h, f G h_{1}\right)=0$ for all $f \in L^{\infty}\left(\mathbf{R}^{n}\right)$ and all $h_{1} \in L^{2}\left(A_{m}\right)$. Let $\left\{e_{j}\right\}$ be a countable dense set in $L^{2}\left(A_{m}\right)$ and define

$$
B_{m}=\bigcup_{j}\left\{x:\left(G e_{j}\right)(x) \neq 0\right\} .
$$

It follows that $h=0$ a.e. on $B_{m}$ so that we can conclude $\left(\tilde{\mathscr{D}}_{m+1}\right)^{\perp} \subset L^{2}\left(\left(A_{m} \cup B_{m}\right)^{c}\right)$. Looking at (3.8) again we see that $\widetilde{\mathscr{D}}_{m+1} \subset L^{2}\left(A_{m} \cup B_{m}\right)$ so $\widetilde{\mathscr{D}}_{m+1}=L^{2}\left(A_{m+1}\right)$ with $A_{m+1}=A_{m} \cup B_{m}$.

We now assume what we will call our working induction hypothesis: for some $l \geqq 1,(3.8)$ holds for all $m$ with $1 \leqq m<l$ and $\widetilde{\mathscr{D}}_{m}=L^{2}\left(A_{m}\right)$ for $m=1,2, \ldots, l$.

Lemma 3.4. Given our working induction hypothesis,

(i) $\phi_{W}^{(m)}(0) \in \tilde{\mathscr{D}}_{m+1}$ if $m<l$.

(ii) Let

$$
\begin{gathered}
f_{W_{1}, \ldots, W_{m+1}}=\sum_{\pi \in S_{m+1}}\left(\mu_{\pi(1)}-W_{\pi(1)}\right) G\left(\mu_{\pi(2)}-W_{\pi(2)}\right) G \ldots \\
\left(\mu_{\pi(m+1)}-W_{\pi(m+1)}\right) \phi_{0},
\end{gathered}
$$

where $\mu_{j}=\left(\phi_{0}, W_{j} \phi_{0}\right)$. Then for $1 \leqq m \leqq l$,

$$
\tilde{\mathscr{D}}_{m}+\operatorname{Ran}_{m+1}=\operatorname{span}\left\{f_{W_{1}, \ldots, W_{m+1}}: W_{j} \in C_{0}^{\infty}\left(\mathbf{R}^{n}\right)\right\}+\tilde{\mathscr{D}}_{m} \text {. }
$$

(iii) For each $\beta>0$ satisfying $\Sigma_{0}(H)<\beta^{2}+\lambda_{0}<\Sigma_{1}(H)$, there is a $k(\beta)<\infty$ (independent of $m$ and $l$ ) such that for $1 \leqq m \leqq l$,

$$
\left\|\left.\rho_{\beta} G \rho_{\beta}^{-1}\langle x\rangle^{-\frac{1}{2}}\right|_{L^{2}\left(A_{m}\right)}\right\| \leqq k(\beta) \text {. }
$$

Proof. According to our definition of $\operatorname{Ran}_{m}, \phi_{W}^{(m)}(0) \in G \operatorname{Ran}_{m}$, so that if $1 \leqq m \leqq l$,

$$
\phi_{W}^{(m)}(0) \in G \operatorname{Ran}_{m} \subset G \mathscr{D}_{m} \subset G \tilde{\mathscr{D}}_{m}=G L^{2}\left(A_{m}\right) .
$$

But if $1 \leqq m<l$, we see from (3.8) that $G L^{2}\left(A_{m}\right) \subset \tilde{\mathscr{D}}_{m+1}$. Since $\phi_{W}^{(0)}(0)=\phi_{0} \in \tilde{\mathscr{D}}_{1}$, (i) follows.

From (i) and (3.3) it follows that if $m \leqq l$,

$$
\left(H+P_{0}-\lambda_{0}\right) \phi_{W}^{(m+1)}(0)=(m+1)\left(\lambda_{W}^{(1)}(0)-W\right) \phi_{W}^{(m)}(0)+g,
$$

where $g \in \tilde{\mathscr{D}}_{m}$, and thus iterating

$$
\left(H+P_{0}-\lambda_{0}\right) \phi_{W}^{(m+1)}(0)=f_{W, W, \ldots, W}+h,
$$

where $h \in \tilde{\mathscr{D}}_{m}$. By "polarization" $\operatorname{span}\left\{f_{\underbrace{}_{m+1}, W, \ldots, W}: W\right.$ real and in $\left.C_{0}^{\infty}\left(\mathbf{R}^{n}\right)\right\}=\operatorname{span}\left\{f_{W_{1}, \ldots, W_{m+1}}: W_{j} \in C_{0}^{\infty}\left(\mathbf{R}^{n}\right)\right\}$. This proves (ii).

According to Corollary 3.2 of [AHS] there is a $k(\beta)<\infty$ such that if $\beta>0$ satisfies $\Sigma_{0}(H)<\beta^{2}+\lambda_{0}<\Sigma_{1}(H)$, we have

$$
k(\beta)\left\|\rho_{\beta}\langle x\rangle^{\frac{1}{2}}\left(H+P_{0}-\lambda_{0}\right) \phi\right\| \geqq\left\|\rho_{\beta} \phi\right\|
$$


for all $\phi \in C_{0}^{\infty}\left(\mathbf{R}^{n}\right)$. Let

$$
\mathscr{D}_{\rho}=\left\{f \in L^{2}\left(\mathbf{R}^{n}\right): \rho_{\gamma} f \in L^{2}\left(\mathbf{R}^{n}\right) \text { for all } \gamma \text { satisfying } \gamma^{2}+\lambda_{0}<\Sigma_{1}(H)\right\} .
$$

We write $f_{j} \stackrel{\mathscr{D}_{\rho}}{\longrightarrow} g$ if $f_{j} \in \mathscr{D}_{\rho}$ for all $j$ and $\left\|\rho_{\gamma}\left(f_{j}-g\right)\right\| \rightarrow 0$ for all $\gamma<\sqrt{\Sigma_{1}(H)-\lambda_{0}}$.

Suppose $f \in \tilde{D}_{1}$ and $B$ is a ball centered at 0 . Then there is a sequence $f_{j} \in \mathscr{D}_{1}$ with $f_{j} \stackrel{\mathscr{D}_{\rho}}{\longrightarrow} \chi_{B} f$. If $\psi_{j}=G f_{j}$, it follows that $\psi_{j} \in \mathscr{D}_{\rho}$ so that we can substitute $\phi=\psi_{j}$ in (3.11) (this requires a limiting argument) to find

$$
k(\beta)\left\|\rho_{\beta}\langle x\rangle^{\frac{1}{2}} f_{j}\right\| \geqq\left\|\rho_{\beta} \psi_{j}\right\| .
$$

Taking $j \rightarrow \infty$ and using Fatou's lemma we learn

$$
k(\beta)\left\|\rho_{\beta}\langle x\rangle^{\frac{1}{2}} \chi_{B} f\right\| \geqq\left\|\rho_{\beta} G \chi_{B} f\right\| .
$$

Now we take $B \uparrow \mathbf{R}^{n}$ and get

$$
k(\beta)\left\|\rho_{\beta}\langle x\rangle^{\frac{1}{2}} f\right\| \geqq\left\|\rho_{\beta} G f\right\|
$$

for all $f \in \tilde{D}_{1}=L^{2}\left(A_{1}\right)$. Replacing $f$ by $\rho_{\beta}^{-1}\langle x\rangle^{-\frac{1}{2}} f$ we obtain (3.10) for $m=1$. Suppose $1<M<l$ and that (3.10) is true for all $m$ satisfying $1 \leqq m<M$. If $f \in \widetilde{\mathscr{D}}_{M}=L^{2}\left(A_{M}\right)$ and $f=0$ outside a ball, then by definition there exist $g_{i} \in \widetilde{\mathscr{D}}_{M-1}$ and $h_{j} \in \operatorname{Ran}_{M}$ so that $g_{j}+h \stackrel{\mathscr{D}_{\rho}}{\longrightarrow} f$. As in the proof of Proposition 3.1, it follows from the hypothesis of the proposition and (3.3) that $h_{j} \in \mathscr{D}_{\rho}$ and $G h_{j} \in \mathscr{D}_{\rho}$. Thus $g_{j} \in \mathscr{D}_{\rho}$, and from the boundedness of $\left.\rho_{\gamma} G \rho_{\gamma}^{-1}\langle x\rangle^{-\frac{1}{2}}\right|_{L^{2}\left(A_{M}-1\right)}$, it follows that $G g_{j} \in \mathscr{D}_{\rho}$. Thus $\psi_{j}=G\left(g_{j}+h_{j}\right) \in \mathscr{D}_{\rho}$ and

$$
k(\beta)\left\|\rho_{\beta}\langle x\rangle^{\frac{1}{2}}\left(H+P_{0}-\lambda_{0}\right) \psi_{j}\right\| \geqq\left\|\rho_{\beta} \psi_{j}\right\| .
$$

Taking $j \rightarrow \infty$ we obtain

$$
k(\beta)\left\|\rho_{\beta}\langle x\rangle^{\frac{1}{2}} f\right\| \geqq\left\|\rho_{\beta} G f\right\|,
$$

and as before this easily extends to all $f \in L^{2}\left(A_{M}\right)$. Hence (iii) of the lemma is proved.

We now continue with our main line of development. It follows from our working induction hypothesis that if $1 \leqq m \leqq l$,

$\tilde{\mathscr{D}}_{m}=\operatorname{closure}\left(\operatorname{span}\left\{f_{1} G f_{2} G \cdots f_{m-1} G f_{m} \phi_{0}: f_{1}, \ldots, f_{m} \in L^{\infty}\left(\mathbf{R}^{n}\right)\right\}+\tilde{\mathscr{D}}_{m-1}\right)$.

In particular,

$$
\psi=\left(\mu_{2}-W_{2}\right) G\left(\mu_{3}-W_{3}\right) G \cdots G\left(\mu_{l+1}-W_{l+1}\right) \phi_{0} \in \tilde{\mathscr{D}}_{l} .
$$

From (iii) of the lemma $\psi \in \mathscr{D}_{\rho}$. Suppose $f_{1} \in L^{\infty}\left(A_{l}^{c}\right)$ and $B$ is a ball centered at 0 . We choose a sequence $W_{1, j} \in C_{0}^{\infty}\left(\mathbf{R}^{n}\right)$ such that $\left\|W_{1, j}-\chi_{B} f_{1}\right\|_{p} \rightarrow 0$ for all $p \in[1, \infty)$. We want to show the $\mathscr{D}_{\rho}$-convergence of $\left(\mu_{1, j}-W_{1, j}\right) G \psi$, where $\mu_{1, j}=\left(\phi_{0}, W_{1, j} \phi_{0}\right)$. For this it is convenient to note that from (iii) of the lemma it follows that $\left.(H+i) \rho_{\gamma} G \rho_{\gamma}^{-1}\langle x\rangle^{-\frac{1}{2}}\right|_{L^{2}\left(A_{l}\right)}$ is bounded. The easy proof will be omitted. Thus by Sobolev's inequality $\rho_{\gamma} G \psi \in L^{q}\left(\mathbf{R}^{n}\right)$ for some $q>2$. Hence the convergence follows by Hölder's inequality. Similarly we conclude that the other permutations in $f_{W_{1}, j} W_{2}, \ldots, W_{l+1}$ converge in $\mathscr{D}_{\rho}$ so that

$$
\psi^{\prime}=\chi_{B} f_{1} G f_{W_{2}, \ldots, W_{l}}
$$


is in $\tilde{\mathscr{D}}_{l+1}$. If we add to $f_{1}$ a function in $L^{\infty}\left(A_{l}\right)$ we are adding a function in $\tilde{\mathscr{D}}_{l}$ to $\psi^{\prime}$ so we can assume that $f_{1}$ above is an arbitrary $L^{\infty}$ function. Given a function $f_{2} \in L^{\infty}\left(A_{l-1}^{c}\right)$ and a sequence $W_{2, j} \in C_{0}^{\infty}\left(\mathbf{R}^{n}\right)$ with $\left\|\langle x\rangle^{-n}\left(W_{2, j}-f_{2}\right)\right\|_{p} \rightarrow 0$ for all $p \in(1, \infty)$ we use an analysis similar to the one just used to show the convergence of $\rho_{\gamma} \chi_{B} f_{1} G f_{W_{2}, j, \ldots, W_{l}}$. We conclude that

$$
\psi^{\prime \prime}=\chi_{B} f_{1} G f_{2} G f_{W_{3}, \ldots, W_{l+1}}
$$

is in $\tilde{\mathscr{D}}_{l+1}$. Again we can replace $f_{2}$ by an arbitrary $L^{\infty}$ function by adding a function in $\mathscr{D}_{l}$ to $\psi^{\prime \prime}$. Continuing in this way we find that $\tilde{\mathscr{D}}_{l+1}$ contains the span of all functions of the form

$$
f_{1} G f_{2} G \cdots f_{l} G f_{l+1} \phi_{0}+h,
$$

where $f_{j} \in L^{\infty}\left(\mathbf{R}^{n}\right)$ and $h \in \mathscr{D}_{l}$. We can calculate the right side of (3.8) with $m=l$ and find that it is given by (3.12) with $m=l+1$. From what we have just shown $\widetilde{\mathscr{D}}_{l+1} \supset$ (right side of (3.12) with $m=l+1$ ). But a glance at the definitions shows $\mathscr{D}_{l+1}$ cannot be any larger and hence (3.8) holds with $m=l$. We have thus shown that (3.8) holds for all $m \geqq 1$ for an increasing sequence of Borel sets $\left\{A_{m}\right\}$. Taking into account (iii) of the lemma one easily finds that if $A=\bigcup_{m=1}^{\infty} A_{m}$,

$$
\left\|\left.\rho_{\beta} G \rho_{\beta}^{-1}\langle x\rangle^{-\frac{1}{2}}\right|_{L^{2}(A)}\right\| \leqq k(\beta) .
$$

We would thus like to show $L^{2}(A)=L^{2}\left(\mathbf{R}^{n}\right)$. This follows from the following argument: let $P$ be an arbitrary polynomial. It follows that for $f, g \in L^{\infty}\left(\mathbf{R}^{n}\right)$,

$$
f P(G) g \phi_{0} \in L^{2}(A) .
$$

But by the functional calculus we can find a sequence

$$
P_{m}(G) \stackrel{s}{\rightarrow} e^{-t\left(H+P_{0}\right)} \quad(\text { for fixed } t>0)
$$

Thus $f e^{-t\left(H+P_{0}\right)} g \phi_{0}=f e^{-t H} g \phi_{0}+c_{t} f \phi_{0} \in L^{2}(A)$. It follows that

$$
f e^{-t H} g \phi_{0} \in L^{2}(A)
$$

for all $f, g \in L^{\infty}\left(\mathbf{R}^{n}\right)$. Since $e^{-t H}$ is "positivity improving" for $t>0$ [RS], it follows that $L^{2}(A)=L^{2}\left(\mathbf{R}^{n}\right)$.

Finally we need the bound for $\left(H+Q-\lambda_{0}\right)^{-1}$ rather than $\left(H+P_{0}-\lambda_{0}\right)^{-1}$ $=G$. This follows (with a different $k(\beta)$ ) from the resolvent equation. Proposition 3.3 is proved.

We now proceed to show that (3.7) cannot hold for all $\psi \in \mathscr{D}(H)$, at least if we make a mild additional assumption.

Proposition 3.5. Suppose $H=-\Delta+V$, where $V$ satisfies (2.1) and (3.1), $\lambda_{0}<\Sigma_{0}(H)$, and $\beta>0$ with $\Sigma_{0}(H)<\beta^{2}+\lambda_{0}<\Sigma_{1}(H)$. Suppose, in addition, that $Q$ is a real function in $C_{0}^{\infty}\left(\mathbf{R}^{n}\right)$ such that $\lambda_{0} \notin \sigma(H+Q)$. Then (3.7) cannot hold for all $\psi \in \mathscr{D}(H)$.

Proof. Let $G_{Q}=\left(H+Q-\lambda_{0}\right)^{-1}$, and pick $\alpha_{0}>0$ with $\alpha_{0}^{2}+\lambda_{0}<\Sigma_{0}(H)$. We will use the fact that

$$
\left\|\rho_{\alpha_{0}} G_{Q} \rho_{\alpha_{0}}^{-1}\right\|<\infty
$$


which follows from the usual analyticity arguments (see [CT] for example) once it is known that $\lambda_{0}$ is not in the essential spectrum of $H_{\alpha_{0}}$ (see [DHSV]). Assume that (3.7) holds for all $\psi \in \mathscr{D}(H)$. Then

$$
\left\|\rho_{\beta} G_{Q} \rho_{\beta}^{-1}\langle x\rangle^{-\frac{1}{2}}\right\|<\infty .
$$

Consider the operator

$$
F(z)=\rho_{\alpha(z)} G_{Q} \rho_{\alpha(z)}^{-1}\langle x\rangle^{-\frac{z}{2}},
$$

where $\alpha(z)=\left(\beta-\alpha_{0}\right) z+\alpha_{0}$. Matrix elements of $F(z)$ between vectors in $C_{0}^{\infty}$ are analytic for $z$ in a neighborhood of the strip $\{z: 0 \leqq \operatorname{Re} z \leqq 1\}$ and bounded in the strip. Hence we can use the three lines lemma (maximum principle) to conclude that

$$
\sup \{\|F(t)\|: t \in[0,1]\}<\infty .
$$

Choose $\varepsilon$ in $\left(0, \beta^{-1} \sqrt{\Sigma_{0}(H)-\lambda_{0}}\right)$ small enough so that $\langle y\rangle^{\varepsilon} v_{i}(y)\left(-\Delta_{i}+1\right)^{-1}$ is bounded for all $i$. We choose $\alpha_{0}$ so that $\alpha(\varepsilon)^{2}+\lambda_{0}=\Sigma_{0}(H)$. We will obtain a contradiction to the statement that $F(\varepsilon)$ is bounded.

The boundedness of $F(\varepsilon)$ is equivalent to

$$
c\left\|\langle x\rangle^{\frac{\varepsilon}{2}} \rho_{\alpha(\varepsilon)}\left(H+Q-\lambda_{0}\right) \psi\right\| \geqq\left\|\rho_{\alpha(\varepsilon)} \psi\right\|
$$

for all $\psi \in \mathscr{D}(H)$, where $c=\|F(\varepsilon)\|$. Let us assume that $\Sigma_{0}(H)<0$. We will indicate the proof for the easier case $\Sigma_{0}(H)=0$ later. It is easily seen that we can find $X \in \mathscr{L} \backslash\left\{\mathbf{R}^{n}\right\}$ so that $\Sigma_{0}(H) \in \sigma_{\mathrm{disc}}\left(H_{X}\right)$ and thus $H_{X}$ has an exponentially decaying eigenfunction $\phi_{X}$ with eigenvalue $\Sigma_{0}(H)$. We assume $\left\|\phi_{X}\right\|=1$. Let $\psi=\rho_{\alpha(\varepsilon)}^{-1} \phi_{X} \otimes u_{X^{\perp}}$. Then according to (3.13),

$$
c\left\|\langle x\rangle^{\frac{\varepsilon}{2}}\left(H+Q+B-\Sigma_{0}(H)\right) \phi_{X} \otimes u_{X^{\perp}}\right\| \geqq\left\|u_{X^{\perp}}\right\|,
$$

where

$$
B=\alpha(\varepsilon)\left(\frac{x}{\langle x\rangle} \cdot D+D \cdot \frac{x}{\langle x\rangle}\right)+\alpha(\varepsilon)^{2} /\langle x\rangle^{2},
$$

and $D=\nabla$. We choose $u_{X^{\perp}} \in C_{0}^{\infty}\left(B_{R(a)}(a)\right)$, where $B_{R(a)}(a) \subset X^{\perp}$ is a ball of radius $R(a)$ centered at $a$. By a scaling argument we can choose $u_{X^{\perp}}$ so that

$$
\begin{aligned}
\left\|\Delta_{X^{\perp}} u_{X^{\perp}}\right\| & \leqq \text { const } / R(a)^{2}, \\
\left\|D u_{X^{\perp}}\right\| & \leqq \text { const } / R(a), \\
\left\|u_{X^{\perp}}\right\| & =1 .
\end{aligned}
$$

The point $a$ will be chosen to tend toward infinity along a fixed line emanating from the origin while we will choose $R(a)=\delta|a|$, where $\delta>0$ but small. The direction of the fixed line and $\delta$ will be chosen presently. The closed set

$$
S=\bigcup_{X_{i} \nsubseteq X} X^{\perp} \cap X_{i}^{\perp}
$$

is a proper subset of $X^{\perp}$ because each $X^{\perp} \cap X_{i}^{\perp}$ appearing is a vector space of dimension strictly less than $\operatorname{dim} X^{\perp}$. (Otherwise $X^{\perp} \cap X_{i}^{\perp}=X^{\perp}$ and $X_{i} \subset X$.) We choose $a$ to lie in $X^{\perp} \backslash S$ so that $\pi_{i} a \neq 0$ for all $i$ with $X_{i} \notin X$. We choose $\delta \in(0,1)$ so that $B_{2 R(a)}(a) \subset X^{\perp} \backslash S$ for $a$ in the chosen direction. We will use the notation 
$x=(y, z)$, where $y \in X$ and $z \in X^{\perp}$. We can choose $\delta$ small enough so that for some $c^{\prime}>0$, if $X_{i} \notin X,|y| \leqq \delta|z|$, and $z \in B_{R(a)}(a)$, then

$$
|x| \leqq c^{\prime}\left|\pi_{i} x\right| \text {. }
$$

Let $\chi_{1}$ be the characteristic function of the set

$$
\left\{x=(y, z):|y|>\delta|z|, z \in B_{R(a)}(a)\right\},
$$

and $\chi_{2}$ be the characteristic function of

$$
\left\{x=(y, z):|y| \leqq \delta|z|, z \in B_{R(a)}(a)\right\} .
$$

We know that $e^{\gamma\langle y\rangle} \phi_{X}(y) \in L^{2}(X)$ for some $\gamma>0$. Let $V_{X}(x)=\sum_{X_{i} \subset X} v_{i}\left(\pi_{i} x\right)$. We estimate

$$
\begin{aligned}
\left\|\langle x\rangle^{\frac{\varepsilon}{2}}\left(V-V_{X}\right) \phi_{X} \otimes u_{X^{\perp}}\right\| \leqq & \text { const } \cdot\left\|\langle x\rangle^{\frac{\varepsilon}{2}} \chi_{1}\left(V-V_{X}\right) e^{-\gamma\langle y\rangle}(-\Delta+1)^{-1}\right\| \\
& + \text { const } \cdot\left\|\langle x\rangle^{\frac{\varepsilon}{2}} \chi_{2}\left(V-V_{X}\right)(-\Delta+1)^{-1}\right\| .
\end{aligned}
$$

The first term on the right is bounded by $e^{-\mu\langle a\rangle}$ for some $\mu>0$ because if $\chi_{1}(x)>0$, $\langle x\rangle^{\frac{\varepsilon}{2}} e^{-\gamma\langle y\rangle} \leqq$ const $\cdot e^{-\mu\langle a\rangle}$. In the second term we write $V-V_{X}=\sum_{X_{i} \ddagger X} v_{i}$, and in the $i^{\text {th }}$ term we estimate for $\chi_{2}(x)>0$,

$$
\begin{aligned}
\langle x\rangle^{\frac{\varepsilon}{2}} & =\langle x\rangle^{-\frac{\varepsilon}{2}}\langle x\rangle^{\varepsilon} \leqq\langle z\rangle^{-\frac{\varepsilon}{2}} \cdot \text { const } \cdot\left\langle\pi_{i} x\right\rangle^{\varepsilon} \\
& \leqq \text { const } \cdot\langle a\rangle^{-\frac{\varepsilon}{2}}\left\langle\pi_{i} x\right\rangle^{\varepsilon}
\end{aligned}
$$

Thus the second term on the right of (3.15) is bounded by const $\cdot\langle a\rangle^{-\frac{\varepsilon}{2}}$. Summarizing, we have for large $a$,

$$
\begin{aligned}
& \left\|\langle x\rangle^{\frac{\varepsilon}{2}}\left(H+Q+B-\Sigma_{0}(H)\right) \phi_{X} \otimes u_{X^{\perp}}\right\| \\
& \leqq \\
& \quad\left\|\langle x\rangle^{\frac{\varepsilon}{2}} \Delta_{X^{\perp}} \phi_{X} \otimes u_{X^{\perp}}\right\|+\text { const } \cdot\left\|\langle x\rangle^{\frac{\varepsilon}{2}} \frac{x}{\langle x\rangle} \cdot D \phi_{X} \otimes u_{X^{\perp}}\right\| \\
& \quad+\text { const } \cdot\langle a\rangle^{-\frac{\varepsilon}{2}} .
\end{aligned}
$$

The second term on the right of (3.16) can be estimated as follows:

$$
\begin{aligned}
\left\|\langle x\rangle^{-\left(1-\frac{\varepsilon}{2}\right)} x \cdot D \phi_{X} \otimes u_{X^{\perp}}\right\| \leqq & \text { const } \cdot\langle a\rangle^{-\left(1-\frac{\varepsilon}{2}\right)}\left(\left\|\left(y \cdot D_{y} \phi_{X}\right) \otimes u_{X^{\perp}}\right\|\right. \\
& \left.+\left\|\phi_{X} \otimes\left(z \cdot D_{z}\right) u_{X^{\perp}}\right\|\right) \\
\leqq & \text { const } \cdot\langle a\rangle^{-\left(1-\frac{\varepsilon}{2}\right)}
\end{aligned}
$$

since

$$
\left\|z \cdot D_{z} u_{X^{\perp}}\right\| \leqq(|a|+R(a))\left\|D_{z} u_{X^{\perp}}\right\| \leqq(|a|+R(a)) \cdot \text { const } / R(a) \leqq \text { const } .
$$

An easy estimate of the first term on the right side of (3.16) then gives

$$
\begin{aligned}
\|\langle x\rangle^{\frac{\varepsilon}{2}} & \left(H+Q+B-\Sigma_{0}(H)\right) \phi_{X} \otimes u_{X^{\perp}} \| \\
& \leqq \text { const } \cdot\left\{\langle a\rangle^{\frac{\varepsilon}{2}}\left\|\Delta_{X^{\perp}} u_{X^{\perp}}\right\|+\langle a\rangle^{-\frac{\varepsilon}{2}}+\langle a\rangle^{-\left(1-\frac{\varepsilon}{2}\right)}\right\} \\
& \leqq \text { const } \cdot\left\{\langle a\rangle^{-\frac{\varepsilon}{2}}+\langle a\rangle^{-\left(1-\frac{\varepsilon}{2}\right)}\right\} .
\end{aligned}
$$

But for large $a$, this contradicts (3.14). 
If $\Sigma_{0}(H)=0$ a simpler argument works. We replace $\phi_{X} \otimes u_{X^{\perp}}$ with $u \in C_{0}^{\infty}\left(B_{R(a)}(a)\right)$, where $B_{R(a)}(a)$ is an open ball in $\mathbf{R}^{n}$. The vector $a$ is chosen to lie outside of $\bigcup_{i} X_{i}^{\perp}$. The rest of the proof is essentially the same.

We summarize what we have learned so far.

Theorem 3.6. Suppose $\lambda_{0}<\Sigma_{0}(H)$ is a non-degenerate eigenvalue of $H$ (where $H=-\Delta+V$ and $V$ satisfies (2.1) and (3.1)). Suppose the corresponding eigenfunction $\phi_{0}$ has abnormal decay so that $\alpha_{\phi_{0}}^{2}+\lambda_{0}>\Sigma_{0}(H)$. Then there exists a real function $W \in C_{0}^{\infty}\left(\mathbf{R}^{n}\right)$ so that the following holds. Let $\phi(t)$ be a smooth family of normalized eigenfunctions of $H+t W$ with $\phi(0)=\phi_{0}$. Then there is a T>0 so that $\phi(t)$ has normal decay for $0<|t|<T$.

As we mentioned in the Introduction, $\lambda_{0}$ in Theorem 3.6 is an embedded eigenvalue of the operator $H_{\alpha}$ if $\Sigma_{0}(H)<\alpha^{2}+\lambda_{0}<\Sigma_{1}(H)$. It follows from Theorem 3.6 itself that $\lambda_{0} \in \sigma_{\text {ess }}\left(H_{\alpha}\right)$ for otherwise the eigenvalue would persist for small $t$. More precisely, a small change in the argument used to prove Proposition 3.5 shows that if $\alpha^{2}+\lambda_{0}>\Sigma_{0}(H)$, and there is an $X \in \mathscr{L}$ such that $\Sigma_{0}(H) \in \sigma_{\text {disc }}\left(H_{X}\right)$ and $\operatorname{dim} X^{\perp}>1$, then $\lambda_{0}$ is in the interior of $\sigma\left(H_{\alpha}\right)$. (For $N$-particle operators with particles moving in $\mathbf{R}^{v}$ with $v \geqq 2$, the essential spectrum of $H_{\alpha}$ is found explicitly in [DHSV].) If no such $X$ exists, for example, if $n=1$, then this type of argument will not work. However, it is probably the case that $\lambda_{0}$ is in the interior of the point spectrum of $H_{\alpha}^{*}($ !). It would be interesting if a perturbation theory could be developed directly for $H_{\alpha}$ from which Theorem 3.6 would follow.

We now state a result which says that in some sense, normal decay is generic. For that purpose let $\mathscr{B}_{1}$ be the set of all real functions $W$ with $|W|_{1}<\infty$. It is easy to see that $\mathscr{B}_{1}$ is a Banach space. Let $\mathscr{B}$ be the closure of the real $C_{0}^{\infty}\left(\mathbf{R}^{n}\right)$ functions in the $|\cdot|_{1}$ norm. The reader can show without difficulty that a real function $W \in \mathscr{B}$ if and only if both $W(-\Delta+1)^{-1}$ and $(-\Delta+1)^{-1}[X \cdot D, \mathrm{~W}](-\Delta+1)^{-1}$ are compact operators.

We agree to call an eigenvalue $\lambda_{0}$ normal if the corresponding eigenfunctions all decay normally, and abnormal otherwise.

Theorem 3.7. Suppose $H=-\Delta+V$, where $V$ satisfies (2.1) and (3.1). Then the set of all $W \in \mathscr{B}$ such that the interval $\left(-\infty, \Sigma_{0}(H)\right)$ contains only normal eigenvalues of $H+W$ is a dense $G_{\delta}$.

Proof. When Theorem 2.6 is referred to in the proof, we mean both Theorem 2.6 and the remark following. Let $\Lambda \subset\left(-\infty, \Sigma_{0}(H)\right)$ be compact and define

$$
\mathcal{O}_{\Lambda}=\{W \in \mathscr{B}: \text { all eigenvalues of } H+W \text { in } \Lambda \text { are normal }\} \text {. }
$$

Suppose $W \in \mathcal{O}_{\Lambda}$. Then there is an open ball $U \subset \mathscr{B}$ centered at the origin so that if $\tilde{W} \in U$, then $H+W+\tilde{W}$ has only normal eigenvalues in $\Lambda$. This follows from Theorem 2.6 and a compactness argument. Thus $\mathcal{O}_{\Lambda}$ is open.

We now show that $\mathcal{O}_{A}$ is dense in $\mathscr{B}$. Given $W \in \mathscr{B} \backslash \mathcal{O}_{\Lambda}$ we will find a sequence $W_{m} \in \mathscr{B}$ with $\left|W_{m}\right|_{1} \rightarrow 0$ so that $W+W_{m} \in \mathcal{O}_{\Lambda}$. To find $W_{m}$ first choose $\widetilde{W}_{m}$ real and in $C_{0}^{\infty}\left(\mathbf{R}^{n}\right)$ such that $\left|\tilde{W}_{m}-W\right|_{1}<\frac{1}{3 m}$, and let $W_{m}^{1}=\tilde{W}_{m}-W$. We now add a real function $W_{m}^{2}$ in $C_{0}^{\infty}\left(\mathbf{R}^{n}\right)$ with $\left|W_{m}^{2}\right|_{1}<\frac{1}{3 m}$ so that all eigenvalues of $H+W+W_{m}^{1}+W_{m}^{2}$ in $\Lambda$ are non-degenerate. Lemma 5.5 in [AHS] is helpful here. The function $W+W_{m}^{1}+W_{m}^{2}$ is real and in $C_{0}^{\infty}\left(\mathbf{R}^{n}\right)$, and thus we can make use of 
Theorem 3.6 where the potential $V$ is replaced by $V+W+W_{m}^{1}+W_{m}^{2}$. Suppose $H+W+W_{m}^{1}+W_{m}^{2}$ has $L$ abnormal eigenvalues in $\Lambda$. Pick one of these eigenvalues and find a real $C_{0}^{\infty}$ perturbation with $|\cdot|_{1}$ norm less than $\frac{1}{(3 m L)}$ which turns this eigenvalue into a normal one. This can be done because of Theorem 3.6. Choose the perturbation small enough so that no eigenvalues enter $\Lambda$. By Theorem 2.6 a small enough perturbation will not increase the number of abnormal eigenvalues in $\Lambda$ so we can proceed one by one to change abnormal to normal eigenvalues. This process adds at most $L$ perturbations with $|\cdot|_{1}$ norm each less than $\frac{1}{(3 m L)}$ so in total a perturbation $W_{m}^{3} \in C_{0}^{\infty}$ with $\left|W_{m}^{3}\right|<\frac{1}{(3 m)}$. Thus $W_{m}=W_{m}^{1}+W_{m}^{2}+W_{m}^{3}$ has $\left|W_{m}\right|_{1}<\frac{1}{m}$.

We have shown that $\mathcal{O}_{A}$ is a dense open subset of $\mathscr{B}$. Choose compact intervals $\Lambda_{m} \subset\left(-\infty, \Sigma_{0}(H)\right)$ so that $\Lambda_{m} \uparrow\left(-\infty, \Sigma_{0}(H)\right)$. Then

$$
G=\bigcap_{m=1}^{\infty} \mathcal{O}_{\Lambda_{m}}
$$

is a $G_{\delta}$, and $W \in G$ if and only if $H+W$ has no abnormal eigenvalues in ( $-\infty$, $\left.\Sigma_{0}(H)\right)$. $G$ is dense by the Baire category theorem.

In Theorem 3.7 we have restricted consideration to the discrete spectrum because in [AHS, Theorems 5.11, 5.11'] it is shown that under mild additional assumptions, generically there are no embedded non-threshold eigenvalues.

We end this section with a discussion of how our methods relate to lower bounds for positive solutions to the Schrödinger equation. First we note that there is an alternative proof of Proposition 3.5 using lower bounds for positive solutions. It goes as follows:

Given the hypotheses we can choose $Q \in C_{0}^{\infty}\left(\mathbf{R}^{n}\right)$ so that $\lambda_{0}<\inf \sigma(H+Q)$ and (3.7) still holds for all $\psi \in \mathscr{D}(H)$. Just use the resolvent equation. But if $f \in C_{0}^{\infty}, f \geqq 0$, and $f \neq 0$, then $\psi=\left(H+Q-\lambda_{0}\right)^{-1} f$ is a positive function satisfying $\left(H-\lambda_{0}\right) \psi=0$ outside a compact set. According to (3.7), $\rho_{\alpha} \psi \in L^{2}$ for some $\alpha>0$ with $\alpha^{2}+\lambda_{0}>\Sigma_{0}(H)$. If certain additional assumptions are made about the $v_{i}$ 's, this contradicts the lower bounds of [CS].

Perhaps more interesting is that Proposition 3.5 can be used to obtain lower bounds of a sort for positive solutions.

Theorem 3.8. Suppose $H=-\Delta+V$, where $V$ satisfies (2.1) and (3.1). Assume, in addition, $V \in K_{n}^{\mathrm{loc}}$, the Kato class described in [AS]. Suppose $\lambda_{0}<\Sigma_{0}(H)$ and that $\psi_{0} \in \mathscr{D}(\Delta)$ satisfies $\left(-\Delta+V-\lambda_{0}\right) \psi_{0}=0$ in $\Omega_{R}=\{x:|x|>R\}$ with $\psi_{0}(x)>0$ in $\Omega_{R}$. Then

$$
\alpha_{\psi_{0}}^{2}+\lambda_{0}=\Sigma_{0}(H)
$$

so that

$$
\lim _{r \rightarrow \infty} \frac{1}{r} \ln \left(\int_{S^{n-1}}\left|\psi_{0}(r \omega)\right|^{2} d \omega\right)^{\frac{1}{2}}=-\sqrt{\Sigma_{0}(H)-\lambda_{0}} .
$$

Proof. We can assume $\lambda_{0}<\inf \sigma(H)$. For if this is not true just add a real $Q \in C_{0}^{\infty}\left(\mathbf{R}^{n}\right)$ so that $\lambda_{0}<\inf \sigma(H+Q)$. We still have $\left(-\Delta+V+Q-\lambda_{0}\right) \psi_{0}=0$ outside a ball. 
We now assume that contrary to the statement of the theorem, $\alpha_{\psi_{0}}^{2}+\lambda_{0}>\Sigma_{0}(H)$. Suppose $f \in C_{0}^{\infty}\left(\mathbf{R}^{n}\right), f$ real and define $\phi$ by

$$
\left(H-\lambda_{0}\right) \phi=f \text {. }
$$

According to [AS], $\phi$ and $\psi_{0}$ are continuous for large $|x|$ and thus by a result of Agmon [A2, Theorem 2.7] for large $|x|$ we have

$$
|\phi(x)| \leqq c_{0} \psi_{0}(x) \text {. }
$$

Thus for some $\alpha>0$ with $\alpha^{2}+\lambda_{0}>\Sigma_{0}(H)$ we have $\rho_{\alpha} \phi \in L^{2}$ for every $\phi$ of the form $\left(H-\lambda_{0}\right)^{-1} f$ with $f \in C_{0}^{\infty}\left(\mathbf{R}^{n}\right)$. Using the kind of approximation argument already used in the proof of Lemma 3.4, it follows that for all $\psi \in \mathscr{D}(H)$,

$$
k\left\|\langle x\rangle^{\frac{1}{2}} \rho_{\alpha}\left(H-\lambda_{0}\right) \psi\right\| \geqq\left\|\rho_{\alpha} \psi\right\| .
$$

But by Proposition 3.5 this is impossible.

\section{Appendix}

We will need an estimate of the form

$$
k\left\|\langle x\rangle^{s} \rho(H+W-\lambda) \phi\right\| \geqq\|\rho \phi\|-\|K \rho \phi\|,
$$

with $\rho=\rho\left(\alpha, \gamma, t, \mu_{1}, \mu_{2}\right)$ given by

$$
\rho(x)=\left(\langle x\rangle /\left(1+\mu_{1}\langle x\rangle\right)\right)^{t} \cdot\left(1+\gamma \mu_{2}\langle x\rangle\right)^{\frac{1}{\mu_{2}}} e^{\alpha\langle x\rangle},
$$

where $\mu_{1} \in[0,1], \mu_{2} \in(0,1], \alpha \geqq 0$, and $K$ is compact. Let $\mathscr{B}_{1}$ be the set of all real-valued measurable functions $W$ on $\mathbf{R}^{n}$ with $|W|_{1}<\infty$. The estimate (A.1) and the conditions for its validity below represent a slight generalization of Theorem 3.1 of [AHS].

Theorem A.1. Suppose $H=-\Delta+V$, where $V$ satisfies (2.1). Fix $t$ and $\alpha_{0}$ nonnegative and suppose $\lambda_{0}+\alpha_{0}^{2} \notin \mathscr{T}(H)$. Then there exist positive contants $\varepsilon, \delta, k$, and a compact operator $K$ so that if $W \in \mathscr{B}_{1},|W|_{1}+\left|\lambda-\lambda_{0}\right|+\left|\alpha-\alpha_{0}\right|<\varepsilon, 0 \leqq \gamma \leqq \delta$, $\mu_{1} \in[0,1]$, and $\mu_{2} \in(0,1]$, then (A.1) holds for all $\phi \in C_{0}^{\infty}\left(\mathbf{R}^{n}\right)$, where $s=1$ if $\alpha_{0}=0$ and $s=\frac{1}{2}$ if $\alpha_{0}>0$.

Proof. The proof in [AHS] suffices with minor variations. The fact that $\alpha(\geqq 0)$ is not fixed above, whereas it is in [AHS, Theorem 3.1] causes no difficulty. For the reader's convenience we present some estimates involving the function $F$ given by $F=\ln \rho$, which are necessary for the proof, and refer the reader to [AHS],

$$
\left(\frac{|x|}{\langle x\rangle}\right) \alpha \leqq|\nabla F| \leqq \alpha+\gamma+t\langle x\rangle^{-1} \text {. }
$$

Define $g$ and $G$ by

$$
\nabla F=x g, \quad G=(x \cdot \nabla)^{2} g-(x \cdot \nabla)|\nabla F|^{2} .
$$

Then if $t, \alpha, \gamma$ are bounded we have

$$
\begin{aligned}
|x \cdot \nabla g| & \leqq c\langle x\rangle^{-1} \\
\alpha\langle x\rangle^{-1} & \leqq g \leqq(\alpha+\gamma)\langle x\rangle^{-1}+t\langle x\rangle^{-2}, \\
G & \leqq c\langle x\rangle^{-1}+4 \gamma(\gamma+\alpha)
\end{aligned}
$$


Theorem A.2. Suppose $H=-\Delta+V$ and $V$ satisfies (2.1). Suppose $\psi \in \mathscr{D}(H)$ and

$$
\left(H-\lambda_{0}\right) \psi=f .
$$

Assume $\langle x\rangle^{t_{1}} e^{\alpha_{1}\langle x\rangle} f \in L^{2}\left(\mathbf{R}^{n}\right)$ for some $t_{1} \geqq 1$ and $\alpha_{1} \geqq 0$. Then

$$
\alpha_{\psi}^{2}+\lambda_{0} \in \mathscr{T}(H) \cup\left[\alpha_{1}^{2}+\lambda_{0}, \infty\right] \text {. }
$$

Suppose in addition that $\alpha_{1}^{2}+\lambda_{0} \notin \mathscr{T}(H)$. Then if $\alpha_{1}=0$ we have $\langle x\rangle^{t_{1}-1} \psi \in L^{2}\left(\mathbf{R}^{n}\right)$, while if $\alpha_{1}>0$ and $\alpha_{\psi} \geqq \alpha_{1}$, we have $\langle x\rangle^{t_{1}-\frac{1}{2}} e^{\alpha_{1}\langle x\rangle} \psi \in L^{2}\left(\mathbf{R}^{n}\right)$.

Proof. We will use (A.1) in the form

$$
k^{\prime}\left\|\langle x\rangle^{s} \rho\left(H-\lambda_{0}\right) \phi\right\| \geqq\|\rho \phi\|-c\|\phi\|,
$$

which follows from (A.1) as (2.2b) was shown to follow from (2.2a). We will also make use of (A.3) for $\phi \in \mathscr{D}(H) \cap \mathscr{D}(\rho)$ instead of just $\phi \in C_{0}^{\infty}\left(\mathbf{R}^{n}\right)$. This follows by a simple approximation agrument. We allow the left side of (A.3) to be infinite.

Suppose that contrary to the statement of the theorem, (A.2) is incorrect. Then $\alpha_{\psi}<\alpha_{1}$ and $\alpha_{\psi}^{2}+\lambda_{0} \notin \mathscr{T}(H)$. We apply Theorem A.1 with $\alpha_{0}=\alpha_{\psi}$. If $\alpha_{\psi}=0$ we choose $\alpha=\alpha_{0}=\alpha_{\psi}=0, \gamma=0$, and $\mu_{1}>0$. Taking $\mu_{1} \downarrow 0$, in (A.3) (with $s=1$ ), we find $\langle x\rangle^{t} \psi \in L^{2}$ for all $t$. A similar argument with $t=0, \alpha=0,0<\gamma<\alpha_{1}, s=1$, taking $\mu_{2} \downarrow 0$, shows that $e^{\gamma\langle x\rangle} \psi \in L^{2}$ for some $\gamma>0$ which contradicts $\alpha_{\psi}=0$. Otherwise $\alpha_{\psi}>0$ so we take $\alpha_{0}=\alpha_{\psi}, 0<\alpha<\alpha_{\psi}$, but $\alpha_{\psi}<\alpha+\gamma<\alpha_{1}$. We set $s=\frac{1}{2}$ and $t=0$. We allow $\mu_{2} \downarrow 0$ which gives $e^{(\alpha+\gamma)<x\rangle} \psi \in L^{2}$, again a contradiction. This establishes (A.2).

To establish the remainder of the theorem we first suppose $\alpha_{1}=0$. Then let $\alpha_{0}=\alpha=\gamma=0, s=1, \mu_{1}>0$. Using (A.3) with $\phi=\psi$ and allowing $\mu_{1} \downarrow 0$ we find that $\langle x\rangle^{t} \psi \in L^{2}$ whenever $\langle x\rangle^{t+1} f \in L^{2}$. If $\alpha_{1}>0$ (and $\alpha_{\psi} \geqq \alpha_{1}$ ) let $\alpha_{0}=\alpha_{1}, 0<\alpha<\alpha_{1}, \alpha+\gamma=\alpha_{1}, \mu_{1}>0, s=\frac{1}{2}$. We can achieve this with $\left|\alpha-\alpha_{0}\right|$ and $\gamma>0$ as small as we please. Substituting $\phi=\psi$ in (A.3) and taking $\mu_{2} \downarrow 0$, $\mu_{1} \downarrow 0$, we obtain $\langle x\rangle^{t} e^{\alpha_{1}\langle x\rangle} \psi \in L^{2}$ whenever $\langle x\rangle^{t+\frac{1}{2}} e^{\alpha_{1}\langle x\rangle} f \in L^{2}$.

\section{References}

[A1] Agmon, S.: Lectures on exponential decay of solutions of second-order elliptic equations. Princeton, NJ: Princeton University Press 1982

[A2] Agmon, S.: Bounds on exponential decay of eigenfunctions of Schrödinger operators. Springer Lecture Notes in Mathematics 1159, ed. S. Graffi, Berlin, Heidelberg, New York: Springer 1985

[AHS] Agmon, S., Herbst, I., Skibsted, E.: Perturbation of embedded eigenvalues in the generalized $N$-body problem. Commun. Math. Phys. 122, 411-438 (1989)

[AS] Aizenmann, M., Simon, B.: Brownian motion and Harnack's inequality for Schrödinger operators. Commun. Pure Appl. Math. 35, 209-273 (1982)

[CT] Combes, J.M., Thomas, L.: Asymptotic behaviour of eigenfunctions for multiparticle Schrödinger operators. Commun. Math. Phys. 34, 251-270 (1973)

[CS] Carmona, R., Simon, B.: Pointwise bounds on eigenfunctions and wave packets in $N$-body quantum systems V. Lower bounds and path integrals. Commun. Math. Phys. 80, 59-98 (1981)

[D1] Derezinski, J.: Exponential bounds in cones for eigenfunctions of $\mathrm{N}$-body Schrödinger operators. Reports on Math. Phys. 24, 219-228 (1986)

[D2] Derezinski, J.: Exponential bounds in cones for eigenfunctions of $N$-body Schrödinger operators II. Ann. Soc. Math. Pol. Comment. Math. 30, 269-275 (1991) 
[DHSV] Deift, P., Hunziker, W., Simon, B., Vock, E.: Pointwise bounds on eigenfunctions and wave packets in $N$-body quantum systems IV. Commun. Math. Phys. 64, 1-34 (1978)

[FH1] Froese, R., Herbst, I.: Exponential bounds and absence of positive eigenvalues for $N$-body Schrödinger operators. Commun. Math. Phys. 87, 429-447 (1982)

[FH2] Froese, R., Herbst, I.: Exponential lower bounds to solutions of the Schrödinger equation: Lower bounds for the spherical average. Commun. Math. Phys. 92, 71-80 (1983)

$\left[\mathrm{FH}_{3} \mathrm{O}_{2}\right]$ Froese, R., Herbst, I., Hoffmann-Ostenhof, M., Hoffmann-Ostenhof, T.: $L^{2}$-exponential lower bounds for solutions to the Schrödinger equation. Commun. Math. Phys. 87, 265-286 (1982)

[Ka] Karner, G.: Many-body systems and the Efimov effect. Few-Body Systems 3, 7-25 (1987)

[K] Kato, T.: Perturbation theory for linear operators. Berlin, Heidelberg, New York: Springer 1966

[KS1] Klaus, M., Simon, B.: Coupling constant thresholds in nonrelativistic quantum mechanics, I. Short range two body case. Ann. Phys. 130, 251-281 (1980)

[KS2] Klaus, M., Simon, B.: Coupling constant thresholds in nonrelativistic quantum mechanics. II. Two cluster thresholds in $\mathrm{N}$-body systems. Commun. Math. Phys. 78, 153-168 (1980)

[P] Perry, P.: Exponential bounds and semifiniteness of point spectrum for $N$-body Schrödinger operators. Commun. Math. Phys. 92, 481-483 (1984)

[RS] Reed, M., Simon, B.: Methods of modern mathematical physics, vol. IV. Analysis of operators. New York: Academic Press 1978

Communicated by B. Simon 